\title{
NavChair: An Example of a Shared-Control System for Assistive Technologies
}

\author{
Simon P. Levine ${ }^{1,3}$, David A. Bell ${ }^{1,2}$, and Yoram Koren ${ }^{2,3}$ \\ Rehabilitation Engineering Program, Dept. of Physical Medicine and Rehabilitation'; \\ Dept. of Mechanical Engineering 2 ; and Graduate Bioengineering Program ${ }^{3}$ \\ University of Michigan
}

\begin{abstract}
The NavChair assistive navigation system was originally conceived as an application of mobile robot obstacle avoidance to a power wheelchair. In this system, the user shares wheelchair control with obstacle avoidance and other navigation components. The philosophy of shared control has important implications for the design of these components. This paper discusses the development of navigation methods for the NavChair guided by design criteria for shared control systems.
\end{abstract}

\section{Introduction}

Human-machine systems in which control of a function is allocated between the user and machine can be termed shared-control systems. Such systems have the potential to achieve desired outcomes which neither the user or machine could achieve independently [18]. Adaptive shared-control systems go one step further by adapting the machine, over time, in response to changing human behavioral characteristics and/or environmental features. Adaptive shared-control systems offer a promise of significantly increased function for individuals who require the use of assistive technologies [11,16].

The NavChair assistive navigation system is being developed to safely improve the mobility of people with motor, sensory, cognitive, and/or perceptual impairments that limit their ability to operate a power wheelchair $[13,8,12]$. The NavChair control system is designed to avoid obstacles, travel safely through doors, and provide other forms of navigation assistance under the direction of the wheelchair user. It is an example of an adaptive shared-control assistive technology system.

The NavChair's shared-control architecture allows users to remain in high-level control while benefiting from the improvements provided by the navigation capabilities [3]. The user indicates the desired direction and speed of travel while various navigation routines modify the user's command, if necessary, to provide improved navigation and safety through a combination of changing the direction of travel and slowing the chair.

Adaptability allows the NavChair to perform well under a wide variety of conditions and is incorporated into the NavChair through the employment of different operating modes. For example, the NavChair has a standard obstacle avoidance mode for operating in open environments, a wall following mode, a door passage mode, and a close approach mode. Some of these are mutually exclusive (i.e. obstacle avoidance and close approach) while others are not (i.e. obstacle avoidance and wall following).

An example of automatic mode selection demonstrates how adaptive shared control can improve system performance. Figure 1 illustrates a situation in which the NavChair performs mode selection in response to a change in user behavior. In this case, environmental variables are not sufficient to determine mode selection 
because the presence of a door doesn't necessarily imply that the user wishes to travel through it. The decision to change modes must be based primarily upon observations of user behavior.
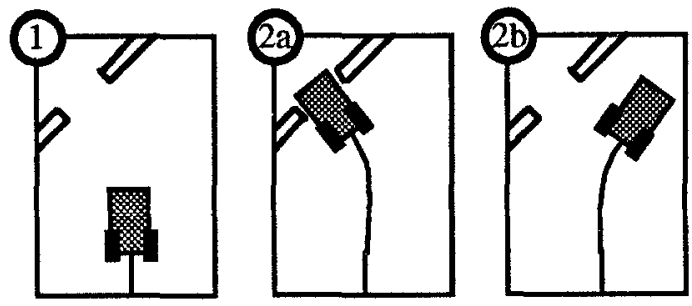

Fig. 1. Mode Selection: Frame (1) shows the NavChair approaching a doorway. One of two outcomes is possible: either (2a) the NavChair performs door-passage behavior, or (2b) the NavChair performs an avoidance maneuver. These two behaviors correspond to two modes of operation, door-passage and obstacle avoidance, that cannot be performed simultaneously.

Two methods are typically used to perform real-time adaptation in human-machine systems: 1) manual user control of machine adaptation where the user selects the NavChair's control mode or 2) automatic machine adaptation based upon observations of environmental variables. Neither of these approaches is fully successful in the NavChair system. Manual mode selection undermines the primary benefits of the NavChair for some of the potential users who do not have the ability to control an additional input. Automatic machine adaptation based only upon environmental feedback is not always successful because it neglects the intention of the user [17].

Our attempts to understand adaptive shared control in the NavChair have lead to general design criteria for adaptive shared-control systems [5]. These criteria include the requirements that adaptive shared-control systems should:

1) model human-machine performance in order to adapt system operation;

2) provide smooth system performance that is stable, comfortable, and intuitive for the user; and

3) include a means for continuously adjusting the degree of influence allocated to the each machine control component vs. the human user.

The first criteria has been satisfied in the NavChair system through the development of a new method, called "Stimulus Response Modeling" (SRM), which is used to automatically determine the most appropriate mode of operation [4]. The primary advantage of SRM over traditional user modeling techniques is that SRM can be applied to the majority of human-machine systems in which user goals can not be measured directly. SRM operates by maintaining a model of user responses to known stimuli and using model parameters as feedback about the user for machine adaptation. Thus, SRM requires that the system must be able to measure disturbances in control loops that involve the user; either measurable external disturbances need to be available for this purpose or the system must be able to apply disturbances that do not interfere with system performance.

Original testing with SRM in the NavChair used a small joystick perturbation (briefly offsetting the resting joystick position from zero) as a stimulus. An autoregressive model relating user joystick responses to these perturbation stimuli was continually updated using recursive system identification $[10,14]$. Stimulus- 
response model parameters provided direct, quantitative information about current user behavior. We have also observed that stimulus-response model parameters reflect changes in user control related to intention. Preliminary results of SRM testing with two subjects operating the NavChair system have demonstrated that a single response can be used to differentiate between driving in an open room vs. a hallway with 80 to $94 \%$ accuracy [4] and that this accuracy increases to nearly $100 \%$ as data is accumulated over time.

In order to provide an effective means for implementing control decisions based on SRM modeling, navigation routines for different modes which satisfied criteria 2 and 3 had to be developed. It became apparent during the development of the NavChair that the obstacle avoidance method initially employed, called the Vector Field Histogram (VFH) method, did not adequately satisfy these requirements as discussed below. This paper describes a new method (MVFH) for assisted navigation in the NavChair system that satisfies these requirements and reports on tests which compares NavChair performance under the control of the VFH vs. MVFH navigation routines.

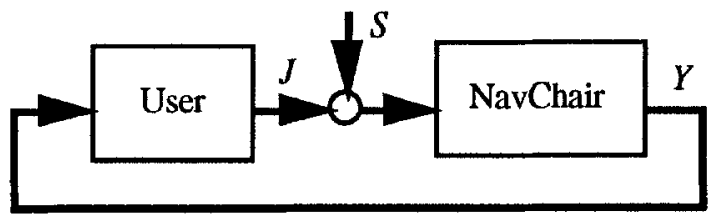

Fig. 2. Stimulus Response Modeling: Observations of responses to an applied stimulus, $S$, are used to model the behavior of the user. The stimulus perturbs the motion of the wheelchair, $Y$, which evokes a response in the joystick command from the user, $J$.

\section{Navigation Methods}

The VFH obstacle avoidance method was originally employed because of its success in autonomous [15] and tele-autonomous robot control [6], which is similar in many ways to the control of the NavChair [4]. A detailed description of the VFH method for this application has previously been presented in the literature [7]. Briefly, VFH performs obstacle avoidance calculations in four steps:

1. Sonar readings are accumulated in a two-dimensional grid that represents the probable locations of obstacles around the wheelchair;

2. Obstacle data is reduced into a polar histogram (h) which is a measure of obstacle density versus direction of travel;

3. The polar histogram is searched for a safe travel direction ( $t$ ) closest to the joystick direction (j)

4. $t$ is modified slightly by an amount proportional to a virtual obstacle repulsive force.

Figure 3 illustrates the behavior of VFH in the scenario from Figure 1. VFH selects a direction of travel $(t)$ that is closest to the desired direction (j) and in which the polar histogram is below the safety threshold value $(\mathrm{m})$. This method allows the NavChair to insure collision-free travel while giving the user high-level control of wheelchair motion. 

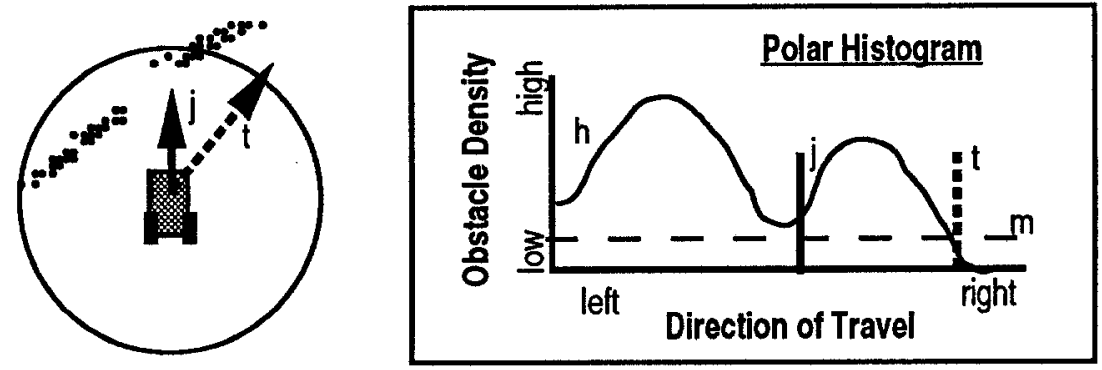

Fig. 3. VFH Obstacle Avoidance in the scenario from Figure 1. The left figure shows the certainty grid around the NavChair; the right figure shows the polar histogram at the same instant, where: $j$ is the desired direction of travel, as indicated by the user with the joystick (solid arrow); $h$ is the polar histogram representing obstacle densities in each possible direction of travel; $m$ is the safety threshold value; $t$ is the actual direction of travel selected by VFH (dotted arrow).

The presence of a human user in the NavChair control loop constrains the design of NavChair components. Design criteria 2 requires that obstacle avoidance provide "intuitive" control for the user. Unintuitive control increases training time and reduces the ability of the user to adapt to unusual circumstances. "Intuitive" control implies, among other things, that the user must feel that the wheelchair's responses to input are rational and predictable. One of the great strengths of the VFH method in autonomous and teleautonomous systems robots is actually a drawback from this standpoint. VFH allows relatively fast travel through cluttered environments by avoiding obstacles with only a minimal reduction in speed. However, this behavior is perceived by a wheelchair user as sudden and unpredictable changes in direction. This perception reflects the invariable degree of influence of VFH; it cannot give the user more or less control in different circumstances. This problem typifies the difficulties experienced in applying robotic obstacle avoidance to the NavChair.

\subsection{MVFH Navigation}

In response to these difficulties, a new navigation method, Minimum VFH (MVFH), was developed that is consistent with our design criteria for shared-control systems. Because it was developed for use in human-machine systems, the design of MVFH provides a broader range of navigation assistance than the obstacle avoidance provided by VFH. MVFH uses the same certainty grid and polar histogram as VFH, but calculates speed and direction of travel differently. MVFH performs navigation calculations in four steps (as illustrated in Figure 4):

1. Sonar readings are accumulated in a two-dimensional grid that represents the probable locations of obstacles around the wheelchair;

2. Obstacle data is reduced into a polar histogram (h) which is a measure of obstacle density versus direction of travel;

3. The sum (s) of the polar histogram (h) and a weighting function (w) is minimized to find a safe direction of travel $(\mathrm{t})$;

4. Wheelchair speed is reduced by an amount that depends on the distance to the nearest obstacle in the path of the wheelchair. 

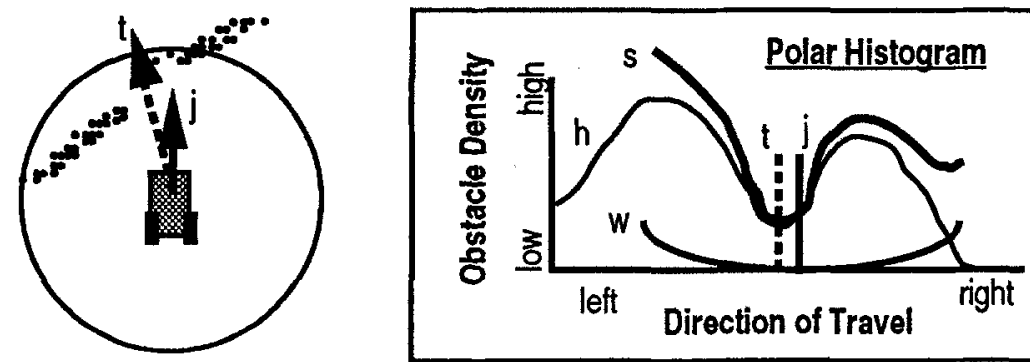

Fig. 4. MVFH Obstacle Avoidance in the scenario from Figure 1. The left figure shows the certainty grid around the NavChair; the right figure shows the polar histogram at the same instant, where: $j$ is the desired direction of travel, as indicated by the user with the joystick (solid arrow); $h$ is the polar histogram representing obstacle densities in each possible direction of travel; $w$ is the weighting function: a parabola centered on the desired direction of travel $(j) ; s$ is the sum of $h$ and $w$; $t$ is the actual direction of travel selected by MVFH at the minimum of $s$.

The first two MVFH steps are identical to those of the VFH method. In step 3, MVFH trades obstacle avoidance against the goals of the user by optimizing a combined function of obstacle avoidance performance and user goals. The shape and steepness of the weighting function, w, controls the degree to which MVFH can influence wheelchair motion: when $w$ is a steep parabola compared to the polar histogram, MVFH can modify the target direction only slightly; when w is relatively flat, MVFH can deviate from the target direction by a large amount, if necessary. Therefore, changing the steepness of $w$ changes the influence of MVFH in providing navigation assistance. With more influence, MVFH causes the wheelchair to go around obstacles with little decrease in speed, while a low degree of influence allows the user to force MVFH to travel close to obstacles and through doors. Therefore, NavChair control modes can be changed by adjusting the steepness and shape of $w$.

Unlike VFH, MVFH does not guarantee that the wheelchair will always move in an obstacle-free direction. Therefore, a collision prevention routine (step 4) slows the chair by an amount proportional to the square root of the distance to the nearest obstacle in the direction of motion. This routine smoothly decelerates the wheelchair to a stop a specified distance from obstacles.

\section{MVFH vs. VFH Testing}

MVFH was compared to VFH by evaluating NavChair performance in terms of the following quantitative measures:

- average speed -- $\mathrm{m} / \mathrm{sec}$.

- jerkiness -- RMS average of the portion of the motor command above $10 \mathrm{~Hz}$.

- average obstacle clearance -- The average distance from the side of the wheelchair to the nearest obstacle.

- collision risk -- collisions and near misses per s.

System performance with obstacle avoidance is related to eight parameters. These obstacle avoidance parameters were set to produce optimal system performance for VFH and MVFH, as measured by the variables above. 


\subsection{Hallway Test}

Five tests were performed in a u-shaped hallway with two right-angle turns. This segment of hallway was selected because it contained difficult trap situations typical of modern buildings: smooth walls, a segment of glass wall, and doors barely wide enough for passage. The course was approximately $30 \mathrm{~m}$ in length and $2 \mathrm{~m}$ wide. Tests 1 and 2 evaluated VFH obstacle avoidance, while tests 3 and 4 used MVFH. In tests 1 and 3, a blindfolded user employed a strategy of pointing the joystick towards one of the walls (at about $45^{\circ}$ ) thereby traveling along that wall while moving down the hallway. In tests 2 and 4, the user's strategy was to point the joystick straight ahead and to travel down the middle of the hall. In all tests, the user was instructed to move the joystick in a different direction if the NavChair stopped moving, such as in corners. In test 5 , an experienced user covered the course as rapidly as possible without obstacle avoidance.

Table 1 compares the results of these tests and shows that MVFH equals or surpasses VFH in terms of every performance measure recorded. In particular, notice that MVFH is as fast as VFH and that its motion is smoother. No collisions occurred using either method. These results suggest that the NavChair system allows a blindfolded person to operate the wheelchair safely at about half the speed of an experienced user.

\begin{tabular}{|c||cc|cc|c|}
\hline $\begin{array}{c}\text { Table 1: } \\
\text { Hallway test number: }\end{array}$ & $\begin{array}{c}\text { VFH } \\
\text { test 1 }\end{array}$ & test 2 & $\begin{array}{l}\text { MVFH } \\
\text { test 3 }\end{array}$ test 4 & $\begin{array}{c}\text { User } \\
\text { test 5 }\end{array}$ \\
\hline \hline speed $(\mathrm{m} / \mathrm{s})$ & 0.73 & 0.78 & 0.77 & 0.78 & 1.62 \\
clearance $(\mathrm{m})$ & 0.44 & $\mathrm{n} / \mathrm{a}$ & 0.45 & $\mathrm{n} / \mathrm{a}$ & $\mathrm{n} / \mathrm{a}$ \\
jerkiness & 0.95 & 0.68 & 0.58 & 0.55 & $\mathrm{n} / \mathrm{a}$ \\
collisions & 0 & 0 & 0 & 0 & 0 \\
\hline
\end{tabular}

Table 1. Four measures of performance are compared for a blindfolded user using obstacle avoidance and an expert user in the smooth hallway course. These results indicate that the blindfolded user is able to travel safely at about half the speed of the experienced user traveling without obstacle avoidance. MVFH slightly outperforms VFH.

\subsection{Door Passage Test}

Figure 6 compares experimental results of door-passage success for the original and Minimum VFH methods. Ten trials were made at door widths from 0.65 to 1.2 meters. The ratio of successful to attempted door passages was recorded for each width. Success was defined as passage without the need for user intervention. MVFH is more successful at door passage than VFH because it allows the NavChair to move closer to obstacles (the doorposts) and because it naturally tends to center the chair as it approaches the doorway.

\section{Discussion}

Many human-machine system components are developed as autonomous machines. However, design criteria for human-machine system components are substantially different than for autonomous systems. An awareness of the differences in design philosophy between autonomous and shared-control systems is necessary 
for the development of the best possible rehabilitation technologies and to allow autonomous components to be integrated into effective human-machine systems.

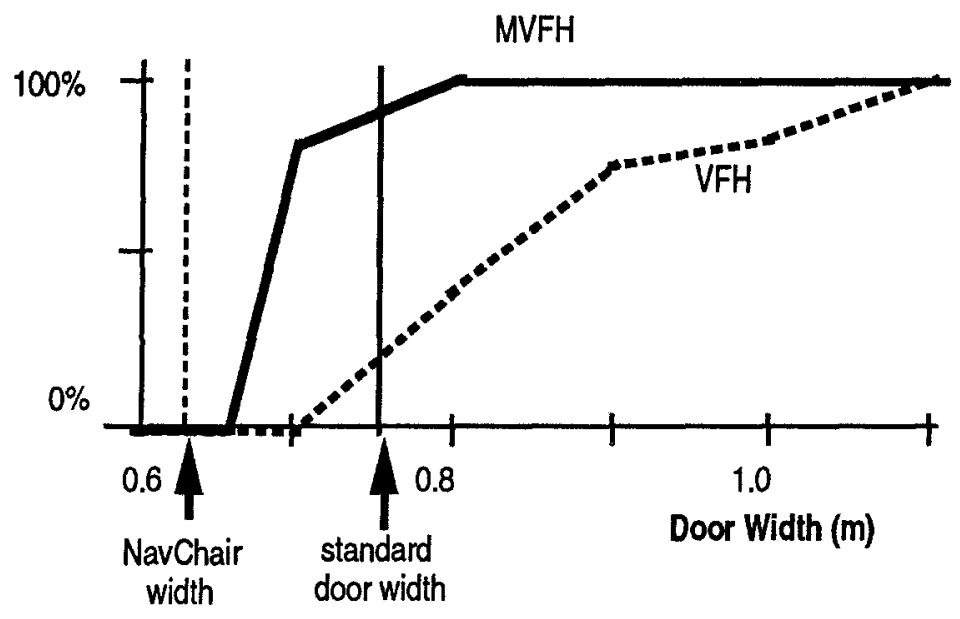

Fig. 6. Door Passage Test Results: Percentage of successful door passage versus door width for VFH (dashes) and MVFH (solid). Two vertical marks provide scale: 1) dashed: the NavChair is $0.63 \mathrm{~m}$ wide; and 2) solid: standard doors are $0.76 \mathrm{~m}$ wide.

The design criteria presented above guided the development of MVFH, a new method for assistive navigation, and SRM, a method of modeling changes in user behavior for adaptive shared control. MVFH provides: 1) relatively fast, safe and effective obstacle avoidance; 2 ) a variable and controllable degree of influence on wheelchair motion; and 3) safe and effective door passage.

SRM allows the full adaptive capabilities of MVFH to be utilized by adapting the influence of MVFH in response to changes in user behavior. SRM models operator behavior in real time for adaptive shared control [4]. Model accuracy increases as data from past times steps is accumulated. For every application, a specific level of accuracy will be required within a specified time to make the implementation of adaptive shared control successful. Rich (i.e., high-power, high-bandwidth) stimuli provide optimal model accuracy, but can negatively impact system performance. Research is being planned to evaluate how stimulus characteristics influence the speed and accuracy of the modeling process. In the NavChair system, we have been able to use the action of MVFH in tests with five subjects as a 'naturally occurring' stimulus that does not degrade system performance at all (in fact, it enhances system performance by providing assistive navigation functions). The MVFH action was found to be a sufficiently rich external stimulus to adequately model the user and thereby allow effective adaptive shared control.

The design criteria presented here were developed in the context of the NavChair system. However, this discussion may benefit other researchers who are experiencing similar difficulties in other human-machine systems. For example, an ability to design systems capable of seamless human-machine adaptive shared control might allow a communication system to automatically configure itself to optimally fit a user's changing abilities or strategy. As another example, an ability to model 
changes in driver behavior in real time could allow a car to adapt the efficiency/performance settings of its transmission to meet the current needs of the driver. These and many human-machine systems stand to benefit from this research in adaptive shared control.

Acknowledgments The NavChair Project is funded under grant B630-DA from the Department of Veteran Affairs Rehabilitation Research and Development.

\section{References}

1. Arai, F., T. Fukuda, Y. Yamamoto, T. Naito, T. Matsui. "Interactive Adaptive Interface Using Recursive Fuzzy Reasoning." IEEE VRAIS 93 1993: 104-110.

2. Bell, D. A., J. Borenstein, S. P. Levine, Y. Koren, and L. A. Jaros. "An Assistive Navigation System for Wheelchairs Based upon Mobile Robot Obstacle Avoidance." 1994 IEEE Conf. on Robotics and Automation 1994.

3. Bell, D. A., S. P. Levine, Y. Koren, L. A. Jaros, and J. Borenstein. "Design Criteria for Obstacle Avoidance in a Shared-Control System." RESNA '94 1994: 581-583.

4. Bell, D. A., S. P. Levine, Y. Koren, L. A. Jaros, and J. Borenstein. "Shared Control of the NavChair Obstacle Avoiding Wheelchair." RESNA 93 1993: 370-372.

5. Bell, D. A., S. P. Levine, Y. Koren, L. Jaros and J. Borenstein. "An Identification Technique for Adaptive Shared-control in Human-Machine Systems." Proc. of the 15th Annual International Conf. of the IEEE Engineering in Medicine and Biology Society 1993: 1299-1300.

6. Borenstein, J., and Y. Koren. "Tele-autonomous Guidance for Mobile Robots." IEEE Transactions on Systems. Man. and Cybernetics 17(4) (1991): 535-539.

7. Borenstein, J., and Y. Koren. "The Vector Field Histogram - Fast Obstacle Avoidance for Mobile Robots." IEEE Journal of Robotics and Automation 1989.

8. Borenstein, J., S. P. Levine, and Y. Koren. "The NavChair--A New Concept in Intelligent Wheelchair Control for People with Multiple Handicaps." CSUN's Fifth Annual Conference on Technology and Persons with Disabilities 1990.

9. Browne, D. "Adaptation and Operator Models" Position Paper for the $\mathrm{CHI}+\mathrm{GI}$ '87 Workshop on Operator Models 1987.

10. Eykhoff, P. System Identification: Parameter and State Estimation. New York: Wiley. 1974.

11. Hancock, P. A., and M. H. Chignell. "Adaptive Control in Human-machine Systems." Ed P. A. Hancock. Human Factors Psychology. New York: Elsevier Science P. 1987. 305-346.

12. Jaros, L. A., D. Bell, S. P. Levine, J. Borenstein, and Y. Koren. "NavChair: Design of an Assistive Navigation System for Wheelchairs." RESNA 93 1993: 379-381.

13. Levine, S., Y. Koren, and J. Borenstein. "NavChair Control System for Automatic Assistive Wheelchair Navigation." RESNA 13th Annual Conference 1990.

14. Ljung, L. Theory and Practice of Recursive Identification. Cambridge, MA: MIT P. 1983.

15. Manz, A., R. Liscano, and D. Greene. "A Comparison of Real-time Obstacle Avoidance Methods for Mobile Robots." Experimental Robotics June, 1991.

16. Schönpflug, W. "Coping Efficiency and Situational Demands." in G. R. J. Hockey, ed., Stress and Fatigue in Human Performance. 1983: 299-329.

17. Schweighardt, M. F. "Using the Context of Interactions to Adapt to Users." Proceedings of the Human Factors Society 34th Annual Meeting_1990: 346-351.

18. Sheridan, T. B. "Telerobotics." Automatica 25(4) (1989): 487-507. 